\title{
Research Paper: A Comparison of Selective Attention Processing in Major Depressive Disorder and Schizophrenia

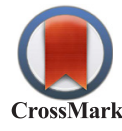

Masoud Fazilat-Pour ${ }^{1 *}$, Latifeh Sharif-Pour ${ }^{1}$, Shahrad Arjmand ${ }^{1}$

1. Department of Psychology, Faculty of Humanities \& Literature, Shahid Bahonar University of Kerman, Kerman, Iran.

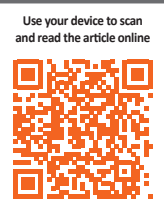

cittat On: Fazilat-Pour, M., Sharif-Pour, L., \& Arjmand, Sh. A Comparison of Selective Attention Processing in Major Depressive Disorder and Schizophreni. Journal of Practice in Clinical Psychology, 5(3), 217-225. https://doi.org/10.18869/acadpub.jpcp.5.3.217

dol: : https://doi.org/10.18869/acadpub.jpcp.5.3.217

Article info:

Received: 12 Feb. 2017

Accepted: 19 May 2017
Keywords:

Attention, Depressive disorder, Major, Schizophrenia

\section{ABSTRACT}

Objective: Cognitive deficits play an important role in differential diagnostics, prognosis and rehabilitation of people with psychological disorders. Attention problems have profound impact on the therapeutic response, risk of relapse, function and quality of life in people with major depressive disorder and schizophrenia. This study compared selective attention of people with schizophrenia, Major Depressive Disorder (MDD), and healthy controls.

Methods: The sample consisted of 16 hospitalized schizophrenics, 16 with major depressive disorder, and 16 matched healthy controls. The Stroop color word task was presented to the participants, and the RTs and error rates in naming the color stimuli were recorded. Data were analyzed by one-way ANOVA and multivariate regressions.

Results: Those in the MDD group and healthy controls showed the highest and the lowest error rates and RT averages, respectively. In addition, the demographic/personal variables were also used to predict the performance measures.

Conclusion: The findings gave support to the notion of morbidity-specific selective attention impairment. The higher levels of interference in the MDD group have been explained in line with the filtration deficit conceptualization.

\section{Introduction}

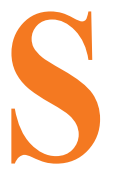

chizophrenia ( $\mathrm{Scz})$ is a complicated and intensive mental disorder affecting more than 21 million people worldwide (World Health Organization, 2017). Attention impairment is the hallmark feature of Scz (Bleuler, 2010), and it is prevalent across the different subtypes of Scz (Liu, Hwu, \& Chen, 1997). Major Depressive Disorder (MDD) is another psychological con- dition with severe impairments in cognitive processing (Trivedi \& Geer, 2014) and deficient selective attention (Mathews \& MacLeod, 2005).

Attention deficits as measured by Stroop Color Word Task (SCWT) are the shared feature of Scz and MDD (Lemelin et al., 1996). Findings showed that schizophrenic symptoms appeared at a later stage compared to MDD, in response towards incongruent stimuli. However, their accuracy rates remained the same

* Corresponding Author:

Masoud Fazilat-Pour, PhD

Address: Department of Psychology, Faculty of Humanities \& Literature, Shahid Bahonar University of Kerman, Kerman, Iran

Tel: +98 (918) 7027800

E-mail: Fazilatm@uk.ac.ir 
(Degl'Innocenti, Ågren, \& Bäckman, 1998). The accuracy rates in MDDs were found to be lower than that of the normal people (Lockwood, Alexopoulos, \& van Gorp, 2002). However, further findings revealed that depressed people showed more delays in answering the incongruent stimuli when compared to Scz group (Lemelin, Baruch, Vincent, Everett \& Vincent, 1997).

Lemelin and colleagues (1997) considered two hypotheses of distractibility/inhibitory deficit and lack of resource allocation as the possible explanations of attention impairments in MDD. They analyzed three interference measures; a typical Stroop interference score, a distractor inhibition score that was the difference between nonconflicting word RT and Color-Word RT; and a Stroop conflict resolution score that was Color-Word RTs minus nonconflicting Word RTs. Part of the results gave support to the notion of inhibitory deficits in MDD (Hertel \& Rude, 1991). In addition, results from the correlational analysis gave further support to resource allocation deficit (Hasher \& Zacks, 1979). Thus, the findings in this context do not offer a persistent picture of speed and accuracy of people's performance.

Siegle, Steinhauer and Thase (2004) showed considerable yet significant differences between the cognitive load in depressed and nondepressed participants. They measured the opening in the pupil of the eye. Depressed people showed lower amounts of pupil dilation in the seconds after the stimulus presentation. Siegle and collaborators attributed this observation to a decreased permanent engagement on the task. Reduction of permanent engagement is a phenomenon that explains the problems related to the reduction in attentional resources and the attentional problems in depressed people. Caprile et al. (2015) addressed the visual attention deficits in Scz only when the task was rule-based or when it required top-down processing. This means they were more likely to show impairments when the stimulus selection was driven by top-down processing.

Desseilles and colleagues (2009) compared the performance of a group of non-medicated unipolar MDDs with matched controls in two tasks manipulated in difficulty levels (easy, difficult) of attention load. The load was on the fixation point in the presence of irrelevant colored stimuli presented in the periphery. Among their findings from the fMRI data, there was abnormal filtering of the irrelevant information in the visual cortex of MDDs. The results showed that the biological abnormalities contributed to the cognitive deficits seen in MDD. Pastò and Burack (2002) compared Sczs with depressed and nondepressed people to measure the filtration of the visual stimuli. They manipulated the presence or absence and the position of distracters plus the presence or the absence of a window cue that should facilitate the concentration of attention in a systematic way. Despite their prediction, Scz and the normal people with no background of psychiatry disorder showed the same performance patterns on the second part of the task that was tapped more into the focal attention processing. The distracter stimuli, which were close to the focal point of attention, showed a negative effect on the people's performance, regardless of the group. But the MDD group showed a better performance in the presence of window cue. From three studied groups, only the performance of people with MDD improved as the result of a cue window. In addition, the ability for filtering and limiting the focus of attention in Sczs was intact. The performance of people with MDD showed a deficiency at filtering and corrected this deficit by the help of a window cue that facilitated the individuals' attention. By this, they claimed for a filtration deficit in MDD.

Further, in a meta-analysis, Stefanopoulou et al. (2009) claimed that differences in the cognitive functioning between MDDs and Sczs are more or less quantitative rather than being qualitative. Deficits in the performance measures were higher for Scz. However, there were differences in the inhibition deficits between those with affective disorders and controls, whereas the performance of Scz and controls were similar. Based on their findings, the only measure for which both the Scz and control group differed was the SCWT, where those with affective disorders showed the highest levels of performance impairments. The interpretation was the possibility of the impaired performance of $\mathrm{Scz}$ in both the congruent and incongruent conditions (Frangou, Dakhil, Landau, \& Kumari, 2006). The other interpretation was that those with Scz had greater sensory and phonological encoding levels. They finished with the claim that response inhibition deficits may be a common cognitive dysfunction in affective disorders despite the diagnosis but not in those with Scz.

It is worth recalling that some studies showed no difference in the selective attention measure between Sczs, MDD, bipolar disorder and those with other psychosis disorders (Verdoux \& Liraud, 2000). Egeland et al. (2003) showed that in terms of processing speed and selective attention, Sczs are lower than the healthy controls. Reichenberg et al. (2002) showed the lowest performance level in Sczs when compared to the patients with schizoaffective disorder, bipolar disorder, and MDDs. Schaub, Neubauer, Mueser, Engel and Möller (2013) compared neuropsychological test profiles in stabilized post-acute inpatients with affective disorders or 
Scz. They showed that patients with depressive disorder are significantly better in verbal and visual short-term memory, verbal fluency, visual-motor coordination, information processing in visual-verbal functioning, and selective attention as compared to Sczs.

Cognitive deficits play a key role in prognosis, differential diagnostics, rehabilitation, and patient's social reintegration (Harvey, 2008). Therefore, identification of morbidity-relevant cognitive impairments among people with different psychiatric diagnostic entities seems vital (Šoštarič \& Zalar, 2011). On the importance of cognitive changes in depression, it is enough to know that such deficits have a profound impact on the therapeutic response, risk of relapse, function and quality of life (Gonda et al., 2015). In addition, traditionally, Scz has been regarded as a primary cognitive disorder (Harvey, 2008). Considering the key role of attentional problems in the psychopathology of MDD and Scz and the inconsistencies in the earlier findings, this study aimed to examine the possible differences in people with Scz, MDD, and healthy controls using the SCWT. To the best of our knowledge, there is no replication study that examined the inhibitory deficits in Iranian sample using the same method.

\section{Methods}

The casual-comparative method was implemented to compare the performance of those with Scz and MDD with healthy controls in the SCWT. The target population included the entire patients hospitalized at Shahid Beheshti Psychiatric Hospital of Kerman city, who were diagnosed with schizophrenia or major depressive disorder. The sample included 48 participants who were selected using convenient sampling method, including 16 schizophrenics and 16 with MDD, with a nearly equal ratio of women and men. Rest 16 with an equal ratio of gender were selected from the healthy population with no background of mental health conditions as the healthy control group. The control group individuals were compared with the patients belonging to the other two groups in terms of gender and educational level. Healthy controls were interviewed by an assistant psychologist according to the criteria of the Statistical Manual of Mental Disorders-Fourth Edition (DSM-IV-TR).

The inclusion criteria included 1) diagnosis with one of the schizophrenia disorders or MDD based on the decision by the clinician expert team that consisted of a psychiatrist and a clinical psychologist, and 2) to have the ability of read and write. The exclusion criteria included comorbidity of schizophrenia and MDD when people could not take part in the study and inability to read and write. The ethical committee of the Psychology Department at the Shahid Bahonar University of Kerman approved the study. APA's Ethical Principles of Psychologists and Code of Conduct were followed in the research. The frequencies and percentages of the demographic variables have been presented for each group (Table 1).

SCWT task was used to measure the selective attention processing of patients. We used the classic Stroop measure (Stroop, 1992). The presentation of word stimuli was simultaneous; the whole word stimuli were presented together on two separate sheets for congruent and incongruent conditions. The order of presentation was counterbalanced for the participants in a random order. Psychometric properties of SCWT were reasonable (MacLeod, 1991). Test-Retest reliability of SCWT was satisfactory in an Iranian sample (Ghadiri, Jazayeri, Ashaeri \& Ghazi Tabatabaie, 2006).

The demographic information of the participants was collected from the records of the inpatient ward. After selecting 30 participants from each group of MDD and Scz, they were asked to provide their consent to participate in the study. Only 16 participants from each of the inpatient groups succeeded till the end of the study. The data collection took place in a room that was prepared in advance in the hospital. The data were collected individually. Each of the participants was shown two cards with the contents of congruent and incongruent stimuli as Stroop task by a psychologist. The total time of the study was around 20 minutes for each participant. The RTs for each of the card sets together with the errors were recorders as the performance indices. Data were analyzed by descriptive and inferential statistics using SPSS 19 for Windows and STATA/SE12.0.

\section{Results}

Recorded error rates and RTs were extracted and analyzed with descriptive and inferential analysis. The difference scores were calculated between RTs for the congruent and incongruent conditions as the Stroop index. In addition, the number of errors was counted across the groups.

\section{Accuracy data}

A between-group ANOVA showed a significant effect of group on the mean of errors, $F(2,45)=68.46$, $\mathrm{P}<0.001$. Scheffe post-hoc comparisons showed that the MDD group had a higher mean of errors $(M M D D=3.63$, $\mathrm{SD}=0.88)$ than the schizophrenia group $(\mathrm{MScs}=1.69$, $\mathrm{SD}=0.79)$ and healthy controls (Mcontrol $=0.44$, $\mathrm{SD}=0.62$ ), $\mathrm{P}<0.0001$ (Table 2). Moreover, there was a 
Table 1. Descriptive or demographic variables across the groups

\begin{tabular}{|c|c|c|c|c|c|c|c|}
\hline \multirow{2}{*}{ Variables } & \multirow{2}{*}{ Levels } & \multicolumn{2}{|c|}{ Healthy Controls } & \multicolumn{2}{|c|}{ MDD } & \multicolumn{2}{|c|}{ Schizophrenia } \\
\hline & & $\%$ & $\mathbf{N}$ & $\%$ & $\mathbf{N}$ & $\%$ & $\mathbf{N}$ \\
\hline \multirow{2}{*}{ Gender } & Female & 34.8 & 8 & 34.8 & 8 & 30.4 & 7 \\
\hline & Male & 32 & 8 & 32 & 8 & 36 & 9 \\
\hline \multirow{2}{*}{ Marital statues } & Single & 48 & 12 & 16 & 4 & 36 & 9 \\
\hline & Married & 17.4 & 4 & 52.2 & 12 & 30.4 & 7 \\
\hline \multirow{6}{*}{ Educational level } & Elementary & 0 & 0 & 100 & 1 & 0 & 0 \\
\hline & Guidance school & 0 & 0 & 75 & 3 & 25 & 1 \\
\hline & Diploma & 0 & 0 & 46.2 & 6 & 53.8 & 7 \\
\hline & Technician & 39.1 & 9 & 26.1 & 6 & 34.8 & 8 \\
\hline & Bachelor & 100 & 5 & 0 & 0 & 0 & 0 \\
\hline & Master & 100 & 2 & 0 & 0 & 0 & 0 \\
\hline \multirow{7}{*}{ Fathers' educational level } & Illiterate & 0 & 0 & 100 & 2 & 0 & 0 \\
\hline & Elementary & 12.5 & 1 & 50 & 4 & 37.5 & 3 \\
\hline & Guidance school & 25 & 2 & 25 & 2 & 50 & 4 \\
\hline & Diploma & 11.8 & 2 & 47.1 & 8 & 41.2 & 7 \\
\hline & Technician & 60 & 3 & 0 & 0 & 40 & 2 \\
\hline & Bachelor & 100 & 7 & 0 & 0 & 0 & 0 \\
\hline & Master & 100 & 1 & 0 & 0 & 0 & 0 \\
\hline \multirow{6}{*}{ Mothers' educational level } & Illiterate & 12.5 & 1 & 62.5 & 5 & 25 & 2 \\
\hline & Elementary & 21.4 & 3 & 35.7 & 5 & 42.9 & 6 \\
\hline & Guidance school & 11.1 & 1 & 44.4 & 4 & 44.4 & 4 \\
\hline & Diploma & 14.3 & 1 & 28.6 & 2 & 57.1 & 4 \\
\hline & Technician & 100 & 6 & 0 & 0 & 0 & 0 \\
\hline & Bachelor & 100 & 4 & 0 & 0 & 0 & 0 \\
\hline \multirow{2}{*}{ Medical history } & No & 72.7 & 16 & 9.1 & 2 & 18.2 & 4 \\
\hline & Yes & 0 & 0 & 53.8 & 14 & 46.2 & 12 \\
\hline \multirow{3}{*}{ Treatment received } & No & 100 & 16 & 0 & 0 & 0 & 0 \\
\hline & Psychiatry & 0 & 0 & 50 & 13 & 50 & 13 \\
\hline & Psychotherapy & 0 & 0 & 50 & 3 & 50 & 3 \\
\hline \multirow{2}{*}{ Family history } & No & 59.3 & 16 & 25.9 & 7 & 14.8 & 4 \\
\hline & Yes & 0 & 0 & 42.9 & 9 & 57.1 & 12 \\
\hline \multirow{4}{*}{ Number of suicidal attempts } & 0 & 48.5 & 16 & 12.1 & 4 & 39.4 & 13 \\
\hline & 1 & 0 & 0 & 75 & 6 & 25 & 2 \\
\hline & 2 & 0 & 0 & 80 & 4 & 20 & 1 \\
\hline & 3 & 0 & 0 & 100 & 2 & 0 & 0 \\
\hline
\end{tabular}


Table 2. Mean (SD) of errors rates and RTs across the groups

\begin{tabular}{cccccc}
\hline Performance Measures & Group & N & Mean & SD \\
\hline Number of errors & Schizophrenia & 16 & 1.69 & 0.79 \\
& MDD & 16 & 3.63 & 0.88 & 0.62 \\
& Healthy control & 16 & 0.44 & 1.52 \\
& Total & 48 & 1.92 & 2.38 \\
RTs & Schizophrenia & 16 & 28.69 & 4.34 \\
& MDD & 16 & 40.25 & 1.84 \\
& Healthy control & 16 & 17.94 & 10.87 \\
\hline
\end{tabular}

significant difference between $\mathrm{Scz}$ group (MScs=1.69, $\mathrm{SD}=0.79$ ) and healthy controls (Mcontrol $=0.44$, $\mathrm{SD}=0.62$ ). The highest error rate was observed in the MDD group compared to Scz group. The lowest errors rates were observed in the healthy controls (Figure 1).

RTs

There was a significant effect of group on the RT averages, $\mathrm{F}(2,45)=275.68, \mathrm{P}<0.001$. Post hoc comparisons using Scheffe post-hoc test showed that the MDD group have higher RT averages (MMDD=40.25, $\mathrm{SD}=4.34$ ) than the $\mathrm{Scz}$ group $(\mathrm{MScz}=28.69, \mathrm{SD}=2.38)$ and healthy controls
(MControl=17.94, $\mathrm{SD}=1.84$ ), $\mathrm{P}<0.0001$. No more significant simple effects were observed. In summary, analysis of the RTs showed that the highest RT averages belonged to the MDD group, followed by the Scz group. The lowest averaged RTs were observed in the healthy controls (Figure 1).

\section{Correlation analysis}

Kendal correlation coefficients were computed to predict the Stroop effect in terms of error numbers and RTs by age, marital status, educational level of the patients, educational level of the parents, medical history, duration of medication, length of hospitalization and the

Table 3. Correlation coefficients between the error rates and RTS with demographics $(\mathrm{N}=48)$

\begin{tabular}{|c|c|c|}
\hline Variables & Errors & RTs \\
\hline Age & 0.20 & 0.14 \\
\hline Marital status & $0.30^{*}$ & $0.30^{*}$ \\
\hline Educational level & $-0.47^{* *}$ & $-0.43^{* *}$ \\
\hline Father's educational level & $-0.43^{* *}$ & $-0.34^{* *}$ \\
\hline Mothers' educational level & $-0.40 * *$ & $-0.34^{* *}$ \\
\hline Medical history & $0.56^{* *}$ & $0.53^{* *}$ \\
\hline Duration of drug use (Week) & $0.41^{* *}$ & $0.45^{* *}$ \\
\hline Time of dagnosis (Week) & $0.38 * *$ & $0.43^{* *}$ \\
\hline Length of hospitalization (Week) & $0.43^{* *}$ & $0.45^{* *}$ \\
\hline Family history & $0.29 *$ & $0.37 * *$ \\
\hline Number of suicidal attempts & $0.58 * *$ & $0.53^{* *}$ \\
\hline
\end{tabular}

* $\mathrm{P} \leq 0.05,{ }^{* *} \mathrm{P} \leq 0.01$ 
Table 4. RMSE, $\mathrm{R}^{2}$ and fit statistics after multivariate regressions

\begin{tabular}{ccccccc}
\hline Equation & $\mathbf{N}$ & Parms & RMSE & $\mathrm{R}^{2}$ & $\mathbf{F}$ & $\mathbf{P}$ \\
\hline Error & 48 & 12 & 0.91 & 0.73 & 8.73 & 0.0001 \\
RTs & 48 & 12 & 5.81 & 0.78 & 11.70 & 0.0001 \\
\hline & & & & PRACTICE AI \\
CINICAL PSCH $\oplus$ LOGY
\end{tabular}

number of suicidal attempts. Except for the age, the other variables showed significant correlations with the errors and RTs. First, there was a significant positive correlation between the marital status and the performance measures. The error rates and RTs were higher in the married people $(\mathrm{P}<0.05)$. In addition, there were significant negative correlations between educational levels of the patients and their parents with error rates and RTs $(\mathrm{P}<0.01)$; the higher was the educational level, the lower were the error rates and RTs. Moreover, there were sig- nificant positive correlations of medical history, duration of medication (in weeks), time of diagnosis (in weeks), length of hospitalization (in weeks), family history and the number of suicidal attempts with the RTs and errors, $(\mathrm{P}<0.01)$, as shown in Table 3 . The highest significant positive correlations were observed between the number of suicidal attempts and the error rates and RTs (Table 3 ).

To test the cumulative predictive values of the age, marital status, educational level, father's educational

Table 5. Model statistics and regression weights after multivariate regressions

\begin{tabular}{|c|c|c|c|c|}
\hline Criterion & Predictor & b & SE & $\mathbf{t}$ \\
\hline \multirow{12}{*}{ Errors } & Age & -0.05 & 0.03 & -1.48 \\
\hline & Marital status & -0.06 & 0.56 & -0.11 \\
\hline & Educational level & 0.15 & 0.21 & 0.74 \\
\hline & Father's educational level & -0.08 & 0.28 & -0.29 \\
\hline & Mothers' educational Level & -0.28 & 0.21 & -1.35 \\
\hline & Medical history & 1.59 & 0.44 & $3.62^{* *}$ \\
\hline & Duration of drug use (Week) & -0.52 & 0.47 & -1.1 \\
\hline & Time of diagnosis (Week) & -0.04 & 0.20 & -0.19 \\
\hline & Length Of hospitalization (Week) & 0.64 & 0.41 & 1.55 \\
\hline & Family history & -0.45 & 0.40 & -1.12 \\
\hline & Number of suicidal attempts & 0.91 & 0.21 & $4.36^{* *}$ \\
\hline & Constant & 2.57 & 1.29 & $2^{*}$ \\
\hline \multirow{12}{*}{ RTs } & Age & -0.54 & 0.22 & $-2.47^{*}$ \\
\hline & Marital statues & 6.30 & 3.57 & 1.77 \\
\hline & Educational level & -0.32 & 1.32 & -0.24 \\
\hline & Father's educational level & -1.50 & 1.80 & -0.84 \\
\hline & Mothers' educational Level & -0.63 & 1.33 & -0.47 \\
\hline & Medical history & 7.67 & 2.80 & $2.74^{* *}$ \\
\hline & Duration of drug use (Week) & 0.97 & 3.01 & 0.32 \\
\hline & Time of diagnosis (Week) & -0.66 & 1.26 & -0.53 \\
\hline & Length Of hospitalization (Week) & 1.07 & 2.64 & 0.4 \\
\hline & Family history & 2.36 & 2.56 & 0.92 \\
\hline & Number of Suicidal attempts & 4.08 & 1.33 & $3.08^{* *}$ \\
\hline & Constant & 32.84 & 8.22 & $4^{* *}$ \\
\hline
\end{tabular}




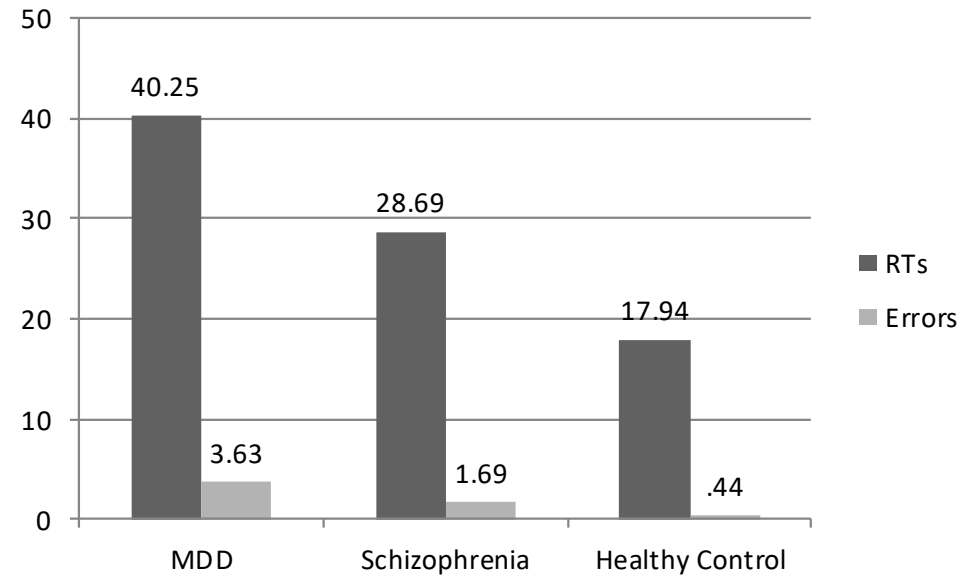

Figure 1. Error and RT averages across the groups

level, mothers' educational level, medical history, duration of drug use (in weeks), time of diagnosis (in weeks), length of hospitalization (in weeks), family history, and number of suicidal attempts, multivariate regressions were employed. As shown in Table 4, the error rates are significantly predicted by predictors, $\mathrm{RMSE}=0.91$, $\mathrm{R}^{2}=0.73, \mathrm{~F}=83.73, \mathrm{P}<0.0001$. Moreover, the equation for the RTs was significant: $\mathrm{RMSE}=5.81, \mathrm{R}^{2}=0.78, \mathrm{~F}=11.70$, $\mathrm{P}<0.00001$. As presented in Table 5 , the medical history showed significant predictive value toward error rates, $b=1.59, t=3.62, P<0.01$, and the number of suicidal attempts, $\mathrm{b}=0.91, \mathrm{t}=4.36, \mathrm{P}<0.01$. RTs were significantly and reversely predicted by age, $\mathrm{b}=-0.54, \mathrm{t}=2.47, \mathrm{P}<0.05$, medical history, $\mathrm{b}=7.67, \mathrm{t}=2.74, \mathrm{P}<0.01$, and the number of suicidal attempts, $b=4.08, t=3.08, P<0.01$. Putting together, the results showed a positive relationship between the medical history and number of suicidal attempts towards the error rates and the similar predictors plus age for the RTs.

\section{Discussion}

This study aimed to compare selective attention processing in people with Scz, MDD and healthy control. The findings showed that the Stroop effect indices as materialized in the error rates and RTs are different across the groups. MDDs showed the greatest latencies, and the lowest latencies were observed in the healthy controls. A similar pattern was observed for the error rates. These findings are in line with the earlier studies that highlighted the attention deficits in MDD (Caprile Cuevas-Esteban, Ochoa, Usall, \& Navarra, 2015; Dest seilles et al., 2009; Frangou et al., 2006; Lemelin et al., 1997; Lockwood et al 2002; Pastò \& Burack, 2002; Ste; fanopoulou et al., 2009). The impaired performance of the MDD group can be explained in terms of the notion of filtration deficit (Pastò \& Burack, 2002). They showed a deficit at filtering irrelevant information. The ability to filter and limit the focus of attention on Scz is relatively intact, and the Scz group can filter the incongruent words-color names better than the MDDs. Depression was found to be associated with reduced distractor inhibition (MacQueen, Galway, Goldberg \& Tipper, 2003).

The findings are differed from the literature in addressing the impaired attentional performance as a shared feature of Scz and MDD (Degl'lnnocenti, Ågren, \& Bäckman, 1998). These studies reviewed the deficits as a shared impairment and a common component of selective information processing in MDD and Scz (Mialet, Pope \& Yurgelun-Todd, 1996; Sereno \& Holzman, 1996). The poorer performance of people with affective disorders is attributed to the psychomotor slowness (Kertzman, et al., 2010) or the resource allocation problems (Hasher \& Zacks, 1979). Depressed people are expected to show impairments where the tasks are tapping more on the attention resources, i.e. in the SCWT (Hasher \& Zacks, 1979).

Another interesting finding was the significant and positive correlations between medical history and the number of suicidal attempts with RTs and error rates; those with medical history showed greater latencies and higher error rates. As shown in the results of the multivariate regressions, the predictors for both the Stroop interference indices (i.e. error rates and RTs) were the same, except for age that was added on top of the medical history and the number of suicidal attempts to predict RTs. This finding is in line with the earlier studies that talk about interference as a vulnerability measure in MDD (Mathews \& MacLeod, 2005). As stated by Gotlib and Cane (1987), depressed patients' performance in the SCWT is different, once while they are in a depressive episode and once again after their recovery from depression. The higher 
was the age level, the greater were the RTs and error rates. In line with the earlier studies (Kertzman, et al., 2010), the age factor played a significant role in predicting the RTs. In summary, when considering the relative importance of the error rates and RTs in predicting the cognitive psychopathology of MDD, it is plausible to think of the observed Stroop performance of MDDs as in a morbidity-specific attention deficits framework. The pattern of the results might be different from the earlier findings (Stefanopoulou, 2009) that claim for quantitative but not qualitative differences in the attention processing of MDD and Scz.

It is worth recalling that the present study has had some limitations; among them is the method of administration of SCWT. For example, Henik and Salo (2004) showed that Scz patients exhibited more interference in the traditional card version of SCWT, i.e., the used version of the present study, and this greater interference level can be explained in terms of their distractibility levels. In contrast, in the other versions of the SCWT, they showed faster RTs to congruent than to neutral trials. In addition, using a single task to claim for attention deficits in MDD and Scz seems insufficient, and other neurocognitive measures should be employed in further studies.

The recent findings from neurological studies support the inhibitory deficit conceptualization (Schaub, Neubauer, Mueser, Engel and Möller, 2013). This indicates that a filtration deficit notion fits better with the pattern of data observed. Future research should talk about questions on underlying mechanisms and focus on a more direct measure of depression-related attentional bias, as mentioned by Epp, Dobson, Dozois and Frewen (2012).

\section{Acknowledgments}

The current study has received no financial support.

\section{Conflict of Interest}

The author declared no conflicts of interest.

\section{References}

Bleuler, E. (2010). Dementia praecox or the group of schizophrenias. Vertex, 21(93), 394-400.

Caprile, C., Cuevas-Esteban, J., Ochoa, S., Usall, J., \& Navarra, J. (2015). Mixing apples with oranges: Visual attention deficits in schizophrenia. Journal of Behavior Therapy and Experimental Psychiatry, 48, 27-32. doi: 10.1016/j.jbtep.2015.01.006

Degl'Innocenti, A., Ågren, H., \& Bäckman, L. (1998). Executive deficits in major depression. Acta Psychiatrica Scandinavica, 97(3), 182-188. doi: 10.1111/j.1600-0447.1998.tb09985.x

Desseilles, M., Balteau, E., Sterpenich, V., Dang-Vu, T. T., Darsaud, A., Vandewalle, G., et al. (2009). Abnormal neural filtering of irrelevant visual information in depression. Journal of Neuroscience, 29(5), 1395-403. doi: 10.1523/jneurosci.3341-08.2009

Egeland, J., Rund, B. R., Sundet, K., Landro, N. I., Asbjornsen, A., Lund, A., et al. (2003). Attention profile in schizophrenia compared with depression: Differential effects of processing speed, selective attention and vigilance. Acta Psychiatrica Scandinavica, 108(4), 276-284. doi:10.1034/j.1600-0447.2003.00146.x

Epp, A. M., Dobson, K. S., Dozois, D. J. A., \& Frewen, P. A. (2012). A systematic meta-analysis of the Stroop task in depression. Clinical Psychology Review, 32(4), 316-328. doi: 10.1016/j.cpr.2012.02.005

Frangou, S., Dakhil, N., Landau, S., \& Kumari, V. (2006). Fronto-temporal function may distinguish bipolar disorder from schizophrenia. Bipolar Disorders, 8(1), 47-55. doi: 10.1111/j.1399-5618.2006.00274.x

Ghadiri F, Jazayeri A, Ashaeri H, Ghazi Tabatabaie M. (2006) [Deficit in executive functioning in patients with schizo-obsessive disorder (Persian)]. Advances in Cognitive Science. 8(3), $11-24$.

Gonda, X., Pompili, M., Serafini, G., Carvalho, A. F., Rihmer, Z \& Dome, P. (2015). The role of cognitive dysfunction in the symptoms and remission from depression. Annals of General Psychiatry, 14(1), 27. doi: 10.1186/s12991-015-0068-9

Gotlib, I. H., \& Cane, D. B. (1987). Construct accessibility and clinical depression: A longitudinal investigation. Journal of Abnormal Psychology, 96(3), 199-204. doi: 10.1037/0021843x.96.3.199

Harvey, P. D. (2008). Cognition and the differential diagnosis of schizophrenia. World Psychiatry, 7(1), 30-32. doi: 10.1002/ j.2051-5545.2008.tb00144.x

Hasher, L., \& Zacks, R. T. (1979). Automatic and effortful processes in memory. Journal of Experimental Psychology: General, 108(3), 356-88. doi: 10.1037/0096-3445.108.3.356

Henik, A., \& Salo, R. (2004). Schizophrenia and the stroop effect. Behavioral and Cognitive Neuroscience Reviews, 3(1), 42-59. doi: $10.1177 / 1534582304263252$

Hertel, P. T., \& Rude, S. S. (1991). Recalling in a state of natural or experimental depression. Cognitive Therapy and Research 15(2), 103-127. doi: 10.1007/bf01173202

Kertzman, S., Reznik, I., Hornik-Lurie, T., Weizman, A., Kotler, M., \& Amital, D. (2010). Stroop performance in major depression: Selective attention impairment or psychomotor slowness? Journal of Affective Disorders, 122(1-2), 167-173. doi: 10.1016/j.jad.2009.08.009

Lemelin, S., Baruch, P., Vincent, A., Everett, J., \& Vincent, P. (1997). Distractibility and processing resource deficit in major depression. Evidence for two deficient attentional processing models. The Journal of Nervous \& Mental Disease, 185(9), 542548. doi: 10.1097/00005053-199709000-00002 
Lemelin, S., Baruch, P., Vincent, A., Laplante, L., Everett, J., \& Vincent, P. (1996). Attention disturbance in clinical depression. Deficient distractor inhibition or processing resource deficit? The Journal of Nervous and Mental Disease, 184(2), 114121. doi: $10.1097 / 00005053-199602000-00010$

Liu, S. K., Hwu, H. G., \& Chen, W. J. (1997). Clinical symptom dimensions and deficits on the Continuous Performance Test in schizophrenia. Schizophrenia Research, 25(3), 211-219. doi: 10.1016/s0920-9964(97)00026-1

Lockwood, K. A., Alexopoulos, G. S., \& Van Gorp, W. G. (2002) Executive dysfunction in geriatric depression. American Journal of Psychiatry, 159(7), 1119-1126. doi: 10.1176/appi. ajp.159.7.1119

MacLeod, C. M. (1991). Half a century of research on the Stroop effect: An integrative review. Psychological Bulletin, 109(2), 163-203. doi: 10.1037/0033-2909.109.2.163

MacQueen, G. M., Galway, T., Goldberg, J. O., \& Tipper, S. P. (2003). Impaired distractor inhibition in patients with schizophrenia on a negative priming task. Psychological Medicine 33(1), 121-9. doi: 10.1017/S0033291702006918

Mathews, A., \& MacLeod, C. (2005). Cognitive vulnerability to emotional disorders. Annual Review of Clinical Psychology, 1(1), 167-195. doi: 10.1146/annurev.clinpsy.1.102803.143916

Mialet, J. P., Pope, H. G., \& YurgelunTodd, D. (1996). Impaired attention in depressive states: A non-specific deficit? Psychological Medicine, 26(5), 1009-1020. doi: 10.1017/ s0033291700035339

Pastò, L., \& Burack, J. A. (2002). Visual filtering and focusing among persons with schizophrenia, major depressive disorder, and no psychiatry history. Canadian Journal of Behavioural Science, 34(4), 239-249. doi: 10.1037/h0087177

Reichenberg, A., Weiser, M., Rabinowitz, J., Caspi, A., Schmeidler, J., Mark, M., et al. (2002). A population-based cohort study of premorbid intellectual, language, and behavioral functioning in patients with schizophrenia, schizoaffective disorder, and nonpsychotic bipolar disorder. American Journal of Psychiatry, 159(12), 2027-2035. doi: 10.1176/appi. ajp.159.12.2027

Schaub, A., Neubauer, N., Mueser, K. T., Engel, R., \& Möller, H.J. (2013). Neuropsychological functioning in inpatients with major depression or schizophrenia. BMC Psychiatry, 13(1), 203. doi: $10.1186 / 1471-244 x-13-203$

Sereno, A. B., \& Holzman, P. S. (1996). Spatial selective attention in schizophrenic, affective disorder, and normal subjects. Schizophrenia Research, 20(1-2), 33-50. doi: 10.1016/09209964(95)00077-1

Siegle, G. J., Steinhauer, S. R., \& Thase, M. E. (2004). Pupillary assessment and computational modeling of the Stroop task in depression. International Journal of Psychophysiology, 52(1) 63-76. doi: 10.1016/j.ijpsycho.2003.12.010

Sostaric, M., \& Zalar, B. (2011). The Overlap of Cognitive Impairment in Depression and Schizophrenia: A Comparative Study. Psychiatria Danubina, 23(3), 251-256. PMID: 21963692

Stefanopoulou, E., Manoharan, A., Landau, S., Geddes, J. R., Goodwin, G., \& Frangou, S. (2009). Cognitive functioning in patients with affective disorders and schizophrenia: A metaanalysis. International Review of Psychiatry, 21(4), 336-356. doi: $10.1080 / 09540260902962149$
Stroop, J. R. (1992). Studies of interference in serial verbal reactions. Journal of Experimental Psychology: General, 121(1), 15-23. doi: 10.1037/0096-3445.121.1.15

Trivedi, M. H., \& Greer, T. L. (2014). Cognitive dysfunction in unipolar depression: Implications for treatment. Journal of Affective Disorders, 152-154, 19-27. doi: 10.1016/j.jad.2013.09.012

Verdoux, H., \& Liraud, F. (2000). Neuropsychological function in subjects with psychotic and affective disorders. Relationship to diagnostic category and duration of illness. European Psychiatry, 15(4), 236-243. doi: 10.1016/s0924-9338(00)00238-8

World Health Organization (2017). Schizophrenia [Internet]. Retrieved from http://www.who.int/mental_health/management/schizophrenia/en/ 